\title{
Why a Non-Metastatic Prostate Cancer Should Become a Castration Resistant Prostate Cancer?
}

\author{
Francesco Carrozza $^{1 *}$ and Matteo Santoni ${ }^{2}$ \\ ${ }^{1}$ Medical Oncology Department of Romagna, Faenza Hospital, Italy \\ ${ }^{2}$ Medical Oncology, Macerata Hospital, Italy
}

*Corresponding author: Francesco Carrozza, Medical Oncology Department of Romagna, Faenza Hospital, 48018, Viale Stradone 9 Faenza (Ravenna), Italy

\section{Short Communication}

The issue of resistance to the "hormonal deprivation control" in Prostate Cancer (PC), is one of the most common and problematic aspects of this disease. It is well known how, under hormonal deprivation, the PC tends to increase over time its threshold of tolerability to ever lower levels of testosterone (usually produced by the cancer itself through paracrine or autocrine mechanisms). The hormone sensitivity (HS) period of PC lasts about 2 years; therefore, the disease subsequently develops increasingly effective mechanisms of resistance to androgen deprivation therapy (ADT), which will transform it into castration-resistant PC (CRPC).

The need for such an adaptive process to be established, however, is generally well justified by the advantage in terms of survival that derives from the beginning of ADT for a metastatic disease. While, in fact, it is clear how ADT can significantly prolong the survival of patients with metastatic PC (mPC), today it is not so evident how ADT itself can contribute to prolong the life of a patient who has not yet measurable metastases, especially in the presence of a slowly rising PSA.

Despite this, however, the number of patients in western countries with a non-metastatic PC (nmPC), previously locally treated, undergoing ADT only for "PSA reasons" is by no means marginal. These patients basically anticipate the time for their PC to become a CRPC. For years, the majority of patients treated definitively for localized PC have started treatments with ADT, or even with complete androgenic blockage (CAB), only because of PSA increases, often even minimal, in the complete absence of secondary lesions documentable with standard diagnostic tools (essentially abdominal/chest CT and bone scan). Furthermore, in many cases, especially in the past, they were patients with low risk PC (GS <8) or extremely long PSA doubling time (PSA DT). Hence, it is reasonable to assume that such patients would not have had a chance of developing a measurable metastatic disease in a short time. The result of this attitude has been to create, over decades of consolidated clinical practice (even if not always supported by scientific evidence), an "artificial" patient population, extremely heterogeneous, and for which it is legitimate to ask: can only the PSA rise after radical PC treatment justify ADT intervention, without measurable metastases? On the other hand, it would no longer be justified to reserve the start of these treatments when the disease recurs clinically, especially considering the recent extremely positive data on the early use of androgen receptor targeting agents (ARTA) in combination with ADT in the metastatic hormone-naive patient $(\mathrm{mHN})$ ?

There is also the question whether the standard diagnostic tools used extensively, especially in the past decades, can really be considered sufficient to fully and comprehensively identify all patients with $\mathrm{mPC}$, especially when the lesions are very small or doubtful (i.e. locoregional lymph nodes $<2 \mathrm{~cm}$ ). The answer to this question is probably no. The use of CT and bone scan, although still considered the diagnostic standard in staging the patient with $\mathrm{mPC}$, is not optimal when it is necessary to find subcentimetric secondary lesions, and therefore many patients judged as nonmetastatic, may result metastatic by the use of updated techniques, such as 11C/18F-Choline or PSMA (Prostate Specific Membrane Antigen) PET. Therefore, defining the nmCRPC setting using PET could be the ideal solution.

Probablyyes, but there is still a problem. Most of the clinical trials that are leading to registration of the new generation antiandrogens in the nmCRPC setting (PROSPER for Enzalutamide, SPARTAN for Apalutamide, ARAMIS for Darolutamide) were carried out using standard diagnostic tools to select patients. The consequence of this is that today we suffer from a not inconsiderable information bias. In other words, the question is: if the patients considered in these trials had been studied with the PET method, would they have actually been "non-metastatic"? Probably not, or at least 
probably not all of them. Furthermore, in many cases, these trials also considered patients with evidence of abdominal lymph nodes $<2 \mathrm{~cm}$ as nmCRPC, which does not make them real "non-metastatic" patients. In summary, today treating a patient defined as nmCRPC according to the above mentioned clinical trials probably means, in most cases, treating an already oligometastatic patient with PET (for which the definition of nmCRPC, therefore, would not be correct). That seems a further proof that we are probably tackling the problem incorrectly.

But let's go back to the initial question: why should a patient become castration resistant without having yet developed PC metastases? The correct answer to this question, in theory, should be only one: because he developed the condition of castration resistance under adjuvant ADT (i.e. after radical prostatectomy, in the presence of $\mathrm{pN}+$ ), or complementary ADT to radiotherapy (both radical EBRT or salvation radiotherapy). Actually, there should be no other ADT application settings outside these areas. Therefore, it is reasonable to conclude that, net of patients belonging to the "adjuvant/complementary" population, nmCRPC patients should not actually exist today. The advent of the multidisciplinary approach will probably lead PC clinicians (primarly urologists, radiotherapists and oncologists) to better select PC patients to be treated with ADT, probably delaying the start of this therapy when it becomes really necessary (i.e. clinical evidence of metastatic disease, and not just for isolated PSA rising). It will therefore be probable to expect in the future an ever-smaller share of PC patients who develop the condition of resistance to castration too early and in the absence of clinically evident metastases.
(C) This work is licensed under Creative

To Submit Your Article Click Here: Submit Article

DOI: 10.32474/RRHOAJ.2020.04.000196

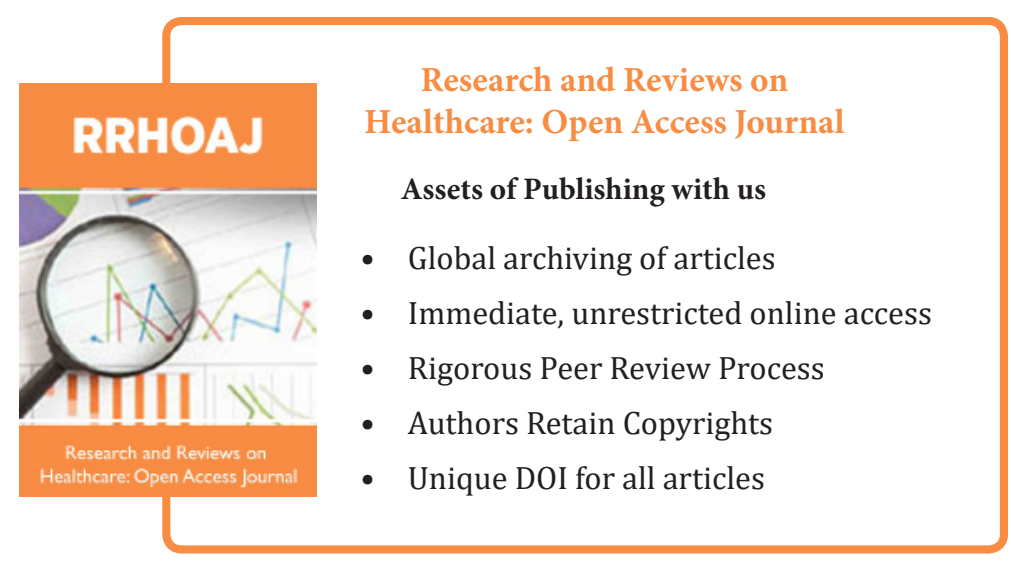

